\title{
Philosophiques
}

\section{Stendhal : " La vérité, l’âpre vérité »}

\section{Patrizia Lombardo}

Volume 40, numéro 1, printemps 2013

\section{Littérature et connaissance}

URI : https://id.erudit.org/iderudit/1018378ar

DOI : https://doi.org/10.7202/1018378ar

Aller au sommaire du numéro

\section{Éditeur(s)}

Société de philosophie du Québec

ISSN

0316-2923 (imprimé)

1492-1391 (numérique)

Découvrir la revue

\section{Citer cet article}

Lombardo, P. (2013). Stendhal : « La vérité, l'âpre vérité ». Philosophiques, 40(1), 87-105. https://doi.org/10.7202/1018378ar

\section{Résumé de l'article}

Stendhal et Musil sont les deux écrivains par excellence qui se sont interrogés sur le type de connaissance qui vient de la littérature. Avant Musil et comme Musil, Stendhal répond à cette question fondamentale en montrant que le roman offre une connaissance des émotions humaines et de leur lien avec les valeurs. Il s'agit à la fois de valeurs éthiques - les situations morales dans lesquelles se trouvent les personnages - et des valeurs esthétiques et proprement littéraires - le tragique, le comique, le tragi-comique, le sublime, etc. Surtout, le roman n'est pas simple représentation du réel, mais aussi du possible. L'analyse de quelques phrases hypothétiques, conjectures et expériences de pensée dans Le Rouge et le Noir, confirme la thèse que la littérature propose une connaissance du possible à travers le travail de l'imagination.
Ce document est protégé par la loi sur le droit d'auteur. L'utilisation des services d'Érudit (y compris la reproduction) est assujettie à sa politique d'utilisation que vous pouvez consulter en ligne.

https://apropos.erudit.org/fr/usagers/politique-dutilisation/ 


\title{
Stendhal: «La vérité, l'âpre vérité»
}

\author{
PATRIZIA LOMBARDO
}

Université de Genève

\begin{abstract}
RÉSUMÉ. - Stendhal et Musil sont les deux écrivains par excellence qui se sont interrogés sur le type de connaissance qui vient de la littérature. Avant Musil et comme Musil, Stendhal répond à cette question fondamentale en montrant que le roman offre une connaissance des émotions humaines et de leur lien avec les valeurs. Il s'agit à la fois de valeurs éthiques - les situations morales dans lesquelles se trouvent les personnages - et des valeurs esthétiques et proprement littéraires - le tragique, le comique, le tragi-comique, le sublime, etc. Surtout, le roman n'est pas simple représentation du réel, mais aussi du possible. L'analyse de quelques phrases hypothétiques, conjectures et expériences de pensée dans Le Rouge et le Noir, confirme la thèse que la littérature propose une connaissance du possible à travers le travail de l'imagination.
\end{abstract}

SUMMARY. - Stendhal and Musil are deeply concerned with the question of the knowledge value of literature. Like Musil and before him, Stendhal answered this question by showing the potential of the novel: this literary form presents human emotions and their connection to values. The characters deal with various situations, therefore convey ethical values, while aesthetic values such as the comic, the tragic, the tragic-comic, the sublime- emerge from the way in which human actions and emotions are represented. All these values are brought about by the style of Stendhal, which is both form and content, both ethical and aesthetic. The analysis of some hypothetical sentences, conjectures and thought experiments in The Red and the Black confirms the thesis later endorsed by Musil, that literature allows for the knowledge of the possible, thanks to the exercise of the imagination.

Mais voici peut-être ce qui est mieux dit: l'homme doué de l'ordinaire sens des réalités ressemble à un poisson qui cherche à happer l'hameçon et ne voit pas la ligne, alors que l'homme doué de ce sens des réalités que l'on peut aussi nommer sens des possibilités traîne une ligne dans

l'eau sans du tout savoir s'il y a une amorce au bout.

Robert Musil, L'Homme sans qualités

Les cinq mots placés en épigraphe du Rouge et le Noir, laconiques et sévères, avec l'article défini et le substantif répétés deux fois, et un seul adjectif, ne laissent pas de doute sur leur signification: la vérité n'est pas suave ou simple. Mais l'âpreté du propos est fêlée par une espièglerie d'écrivain: «La vérité, l'âpre vérité", ces paroles, qui semblent sorties de la bouche de la Pythie, sont paradoxales, car Danton, que Stendhal désigne comme leur auteur, ne 
les a jamais prononcées ni écrites. C'est une fausse citation ${ }^{1}$. Ce clin d'œil ironique dans l'épigraphe même ne sert-il pas à signaler que le roman vise la vérité à travers le faux ou plutôt la fiction, à travers des conjectures qui pourraient être vraies ou qui tirent du réel des variantes possibles? Stendhal suggère ainsi que Danton aurait pu dire ces mots, même s'il ne l'a pas fait. Surtout, comme un coup de fouet, cette sentence concise et rapide projette ce à quoi l'écrivain tend par son œuvre: la littérature comme connaissance, comme recherche de la vérité, et d'une vérité qui n'est évidemment pas celle de l'opposition logique "vrai / faux» (puisqu'une fausse attribution dit vrai sur le possible). Quelles sont les vérités qui ne répondent pas à cette opposition? Ce sont les vérités morales: un programme éthique est donc contenu dans cette épigraphe. Mais aussi un programme esthétique, car cette épigraphe ne correspond pas non plus à l'association entre le vrai et le réel typique du réalisme littéraire, qui semblerait affirmé par la phrase: «Le roman, c'est un miroir que l'on promène le long du chemin ", phrase que Stendhal, dans une autre «fausse » épigraphe du Rouge et le Noir, attribue à Saint-Réal. Ce nom n'est pas en effet celui de l'abbé historien et critique du XVII e siècle, mais un calembour qui signifie ironiquement «saint réel $»^{2}$; la sainteté du réel selon Stendhal n'est pas celle qui sera le fondement du réalisme littéraire, qui a explosé après $\mathrm{I} 850$ et couronné $a$ posteriori Balzac comme écrivain réaliste.

Stendhal et Musil sont les deux écrivains par excellence qui se sont interrogés sur le type de connaissance qui vient de la littérature. À la distance d'un siècle, ils ont posé les mêmes questions et ont donné des réponses presque identiques. Si l'on se demande ce que l'on connaît, inévitablement on se demande aussi comment connaître.

On veut montrer ici que, avant Musil, Stendhal fait comprendre que le roman offre une vision sur les vérités éthiques - donc liées aux valeurs morales et aux émotions humaines. Le roman propose en même temps un choix sur les valeurs esthétiques et proprement littéraires - tragique, comique, tragi-comique, réaliste, romantique, etc. Le roman ne peut convoquer tout cela que par le style, qui sera donc à la fois forme et contenu, à la fois élément éthique et esthétique: ce style n'est pas ornement, mais style de pensée qui fait la manière dont la matière du roman est organisée dans l'agencement des paragraphes, dans la phrase, par le choix des termes, dans la construction des descriptions, dialogues, monologues et commentaires.

\section{Connaissance de la vie}

La vérité chez Stendhal est une valeur cognitive, et non pas sociologique ou historique. Stendhal, familier de la philosophie matérialiste du XVIII ${ }^{\mathrm{e}}$ siècle,

1. Aussi fausse que les petites incohérences de dates à l'intérieur même du roman.

2. G. C. Jones, «Réel, Saint-Réal: une épigraphe du Rouge et le réalisme stendhalien », Stendhal Club, 1983, vol. 25/98, p. 235-243. 
parle souvent de l'importance des faits et de l'observation, et il pense que ce qui est vrai est adéquat à la réalité, mais non pas en coïncidence exacte avec les faits. La littérature pour lui n'est pas un document qui correspond à des faits, ainsi que le veut la thèse positiviste-réaliste, celle que Balzac, en dépit de son aspect visionnaire, affiche dans son célèbre avant-propos de La Comédie Humaine, en I 842, l'année même de la mort de Stendhal:

La Société française allait être l'historien, je ne devais être que le secrétaire. En dressant l'inventaire des vices et des vertus, en rassemblant les principaux faits des passions, en peignant les caractères, en choisissant les événements principaux de la Société, en composant des types par la réunion des traits de plusieurs caractères homogènes, peut-être pouvais-je arriver à écrire l'histoire oubliée par tant d'historiens, celle des mœurs ${ }^{3}$.

Reprenons l'écart entre le sens «vrai» de l'épigraphe du Rouge et le Noir et la "fausseté » de la citation de Danton: c'est l'écart entre des vérités essentielles et des vérités contingentes, de détail — la liste de l'inventaire. Le réalisme littéraire, avec les descriptions riches et pittoresques de Balzac et, plus tard, les descriptions statiques et sensorielles de Zola, est condamné aux détails.

Le roman que veut Stendhal ne cherche pas à rendre les détails, mais traite des vérités essentielles, c'est-à-dire, des questions importantes de la vie, et pose le problème ancien de la meilleure manière de vivre. Les vérités de détail ne comptent pas. Il n'y a que les cuistres — mais, hélas, ils sont nombreux - qui peuvent être choqués par la fausseté de la citation du Ronge et le Noir; seuls ceux qui ne comprennent pas la valeur de l'ironie peuvent entendre les mots au premier degré: âmes sèches, dirait Stendhal, myopie du réalisme pointilleux, ou encore, comme le dirait Robert Musil, petite bureaucratie de la précision. Car il y a une bonne précision qui se bat contre l'imprécision et mène à la clarté, et une mauvaise précision qui cumule les détails ennuyeux et inutiles.

La tension entre les vérités essentielles et les vérités de détail constitue déjà un objet de débat à l'époque romantique. Les romantiques d'école confondent la vérité historique et la vérité cognitive, annoncent l'importance de la couleur locale, habillent le drame et le roman avec le faux-semblant de la réalité, la reconstitution de la contingence par les costumes et le décor - d'où la volupté descriptive de l'époque, comme si rendre l'aspect extérieur du monde, le lieu et l'heure de l'événement était la voie la plus directe vers l'histoire telle qu'elle s'est passée. Il s'agit d'écrivains qui, pour utiliser la comparaison de Musil dans l'épigraphe de cet article, ne voient que l'hameçon.

3. Balzac, «Avant-propos », La Comédie humaine I, Paris, Gallimard, «Bibliothèque de la Pléiade», I97I, p. I I. 
Victor Hugo lance son manifeste dans la préface de Cromwell en I 827:

On commence à comprendre de nos jours que la localité exacte est un des premiers éléments de la réalité. Les personnages parlants ou agissants ne sont pas les seuls qui gravent dans l'esprit du spectateur la fidèle empreinte des faits. Le lieu où telle catastrophe s'est passée en devient un témoin terrible et inséparable; et l'absence de cette sorte de personnage muet décomplèterait dans le drame les plus grandes scènes de l'histoire. Le poëte oserait-il assassiner Rizzio ailleurs que dans la chambre de Marie Stuart? poignarder Henri IV ailleurs que dans cette rue de la Ferronnerie, tout obstruée de haquets et de voitures? brûler Jeanne d'Arc autre part que dans le Vieux-Marché?

Mais Alfred de Vigny est plus nuancé:

Le jour où l'homme a raconté sa vie à l'homme, l'Histoire est née. Mais à quoi bon la mémoire des faits véritables, si ce n'est à servir d'exemple de bien ou de mal? Or les exemples que présente la succession lente des événements sont épars et incomplets; il leur manque toujours un enchaînement palpable et visible qui puisse amener sans divergences à une conclusion morale $[\ldots]^{4}$.

La vérité dans l'art ne saurait être réduite à la couleur locale exaltée par Hugo: pour ce dernier, l'empreinte des faits suffit, pour l'autre, l'intelligence seule permet de relier les faits éparpillés et de leur donner un sens.

Vigny ébauche dans le langage téléologique du XIX ${ }^{\mathrm{e}}$ siècle le principe que j'appellerai: le haut degré d'intensité de prémisses et de conclusions, la haute densité d'inférence dans la fiction littéraire. Ce que dit Vigny est proche de ce que pensent aujourd'hui certains philosophes, tel Gregory Currie, qui parlent de la richesse de la fiction par rapport au monde réel, de sa capacité à rendre palpable ce que Vigny appellerait: l'enchaînement des événements et des situations. Le haut niveau d'inférence de la fiction est le produit de la composition de l'écrivain qui ordonne et organise le réseau d'événements et de motifs, et Currie n'hésite pas à suggérer la comparaison avec la maîtrise du monde qu'aurait un dieu rationnel.

But any coherent narrative, no matter how unobvious the inferences it warrants, allows for vastly more inferential connections between actions, events and Character to be made than is ever legitimate when we are considering the actions and sufferings of real people. We know that the narrative is the product of an overarching intelligence that makes the connections for reasons; so events that in the real world would be connected in a brute causal way will now also be connected by reason and rationality. The closest real-world approximation to this is the inferential practice of someone who thinks the

4. .Victor Hugo, «Préface de Cromwell», Théâtre complet I, Paris, Gallimard, Bibliothèque de la Pléiade, 1963, p. 459.

Alfred de Vigny, «Réflexions sur la vérité dans l'art », Cinq Mars, CEuvres Complètes II, Paris, Gallimard, «Bibliothèque de la Pléiade », I948, p. 20. 
world produced and sustained by an intelligible, rational deity whose aims can be inferred from events down to the level of transactions between individuals ${ }^{5}$.

Vigny parle de morale: les vérités essentielles dans la littérature ont affaire avec les grands thèmes moraux. Comment penser la littérature sans les questions qui l'ont occupée depuis toujours? Les écrivains de tout temps et lieux traitent du bien et du mal — de tout ce qui est humain, trop humain comme Milton, Blake, Melville, Dostoïevski, etc. La liste est longue, car, comme le remarque T. S. Eliot à propos de Baudelaire: "All first-rate poetry is occupied with morality: this is the lesson of Baudelaire. More than any poet of his time, Baudelaire was aware of what most mattered: the problem of good and evil ${ }^{6}$.» Mais la littérature est aussi et surtout concentrée sur des thèmes de morale pratique: comment agir, comment se comporter dans une situation, qu'est-ce qu'une émotion, comment peut-on réfléchir sur les émotions, quelles sont les raisons et les effets d'un sentiment et d'une action? On a beau dire, le livre sur rien dont parlait Flaubert au moment où il écrivait Madame Bovary n'existe pas: un roman est toujours sur quelque chose ${ }^{7}$.

Dans un essai de I9 8 , Musil disait que la littérature offre une connaissance non des faits scientifiques, c'est-à-dire, relatifs à la science ou à la psychologie en tant que science, "mais des motifs qui président aux faits éthiques». Stendhal avait anticipé cette vision. Musil distinguait deux domaines qu'il appelait le «ratioïde» et le "non-ratioïde». Le premier terme indique «ce qui peut entrer dans un système scientifique", et son domaine "est régi par la notion de solidité comme d'une fictio cum fundamento in re (en profondeur les assises sont chancelantes, mais on espère y mettre de l'ordre); domaine de la règle avec exceptions ». Dans le "non-ratioïde», en revanche, les exceptions sont plus importantes que la règle: «il est le domaine des réactions de l'individu au monde et à autrui, des valeurs et des évaluations, des relations éthiques et esthétiques; faits infinis et incalculables...» Le «ratioïde» comporte des constantes, des lois, tandis que le «non-ratioïde» s'ouvre à mille variables. Tout en marquant la différence entre les deux domaines, Musil affirme que le travail de l'écrivain est le même que celui de l'homme de science: il est engagé dans la recherche de la vérité. Sa position est celle de l'investigation: "La tâche de l'écrivain consiste à découvrir sans cesse de nouvelles constellations, de nouvelles variables, à établir des prototypes de déroulement d'événements, des images séduisantes des possibilités d'être un homme,

5. Gregory Currie et Jon Judideini, «Art and delusion», Monist, 86 (2005), p. 556-78.

6. T.S. Eliot, "The Lesson of Baudelaire», The Tyro v. I, Spring I92 I.

7. «Ce qui me semble beau, ce que je voudrais faire, c'est un livre sur rien, un livre sans attache extérieure, qui se tiendrait de lui-même par la force même de son style [...]». (Gustave Flaubert, Correspondance II [Lettre à Louise Colet I 6 janvier I 8 52], Paris, Gallimard, «Bibliothèque de la Pléiade », I980, p. 3 I.) 
d'inventer l'homme intérieur ${ }^{8}$.» Il continue en demandant pourquoi il faut examiner les problèmes éthiques et les émotions dans une œuvre littéraire et non pas dans un traité. En psychologie, qui est une science, et appartient donc au domaine ratioïde, "la diversité des faits n'est nullement infinie ». En revanche: "Ce qui est d'une diversité incalculable, ce sont les motifs de l'âme avec lesquels la psychologie n'a rien à faire.»

L'épigraphe du Rouge et Le Noir pose un problème philosophique, résume une visée dans le savoir qui est chère à Stendhal et dont la forme romanesque devient le moyen privilégié pour la mettre à l'œuvre. Comme Musil, Stendhal pense que la littérature élargit la connaissance de la vie, des affects, des raisons qui agitent le cœur et le cerveau des êtres humains et qui poussent ceux-ci à agir ou ne pas agir (ce qui est aussi un type d'action). On peut ajouter que le roman tente de répondre à la question: quelle vie est la bonne vie? La réponse de Stendhal, même s'il semble négliger la philosophie antique, n'est pas loin de celle de l'Éthique à Nicomaque. Aristote dit qu'il est inutile de parler de Bien suprême comme Platon, car les êtres humains cherchent le bonheur dans l'existence de tous les jours, ou le moins possible de malheur, et les âmes nobles sont celles qui peuvent s'approcher le plus de la bonne vie et du bonheur.

La question du bonheur est centrale pour Stendhal. Le Rouge et le Noir est à la fois roman historique, histoire d'amour, roman de formation et peinture sociale. Le sous-titre donné par Stendhal, Chronique du XIXe siècle, obéit au goût de l'époque pour les récits historiques. Comme dans un roman picaresque ou dans les romans de mœurs du XVIII ${ }^{e}$ siècle - par exemple dans l'Histoire de Tom Jones, enfant trouvé de Henry Fielding, un des écrivains que Stendhal adore - , le protagoniste se fraye sa voie dans le monde, en passant d'une aventure à l'autre. Julien Sorel, fils d'un paysan, parvient à monter dans l'échelle sociale pendant les dernières années de la Restauration; une petite ville du Jura, le séminaire de Besançon, la venue à Paris comme secrétaire du marquis de la Mole, enfin le mariage avec la fille de ce dernier constituent les étapes de son ascension jusqu'à la chute finale, la prison et l'échafaud après le coup de pistolet tiré contre son ancienne maîtresse. Comme dans les récits romanesques anglais (romances), l'amour joue un rôle essentiel. Les personnages principaux, $\mathrm{M}^{\mathrm{me}}$ de Rênal, Mathilde de la Mole et Julien, vivent l'amour-passion avec ses hauts et ses bas, le doute, l'illusion, l'amertume. Mais, contre toutes les attentes du genre, c'est au moment le plus tragique que l'expérience du sublime intervient, durant le séjour du héros en prison, avant d'être guillotiné, car c'est là qu'il connaît le bonheur: il va vers la mort heureux, car il comprend enfin qu'il aime $\mathbf{M}^{\mathrm{me}} \mathrm{de}$ Rênal.

8. Musil, «La connaissance chez l'écrivain. Esquisse », Essais, trad. de Philippe Jaccottet, Paris, Seuil, I978, p. 83. 
Tout lecteur du Ronge et le Noir sait que, dans ce roman, contrairement à beaucoup de littérature de l'époque toute concentrée sur l'amour, ou sur les grandes passions politiques, ou sur une seule passion (comme chez Balzac), on trouve une représentation très riche et variée des sentiments humains. La vanité, l'ambition et l'amour apparaissent dans mille nuances de type et de circonstances, et la liste des émotions serait longue: remords, rage sociale, orgueil, humiliation, pudeur, surprise, gaieté, tristesse, selfdeception, c'est-à-dire erreur ou illusion sur les sentiments qu'on éprouve, tout y est, comme dans tous les romans de Stendhal.

On le voit déjà dans le résumé: Le Rouge et le Noir représente un monde, une époque et une société, mais surtout parle des émotions qui, elles, ne sauraient être séparées des idées, des pensées et des comportements des personnages. Comment Stendhal rend-il compte de cette vie affective? C'est en répondant à cette question qu'on voit mieux la valeur cognitive de la littérature selon Stendhal, et selon tous ceux qui donnent à la littérature une place dans l'entreprise générale de la connaissance. (J'utilise ici les termes de littérature et de roman indifféremment. La littérature comprend beaucoup d'autres formes, mais les limites sont données ici par les deux écrivains que j'associe, Stendhal et Musil, lesquels ont posé le problème de la connaissance à travers la forme du roman — ou du drame, car Stendhal a voulu d'abord écrire des drames, et Musil a écrit des pièces de théâtre. On laissera donc de côté la forme poétique.)

Dans ses romans, Stendhal développe, comme le fera plus tard Musil, une véritable théorie des émotions. Celle-ci peut être résumée en quelques points, lesquels correspondent aux idées du traité sur le sentiment écrit par le personnage d'Ulrich dans L'Homme sans qualités. Une émotion est pour ainsi dire stimulée par les événements, qui, à leur tour, sont influencés par les réponses émotives des individus dans un va-et-vient continu entre l'extérieur et l'intérieur. Les émotions sont nombreuses, variées, toujours en transformation. Une émotion apparaît dans les gestes, les expressions du visage et du corps, et dans les mots. Les émotions ne sont pas ennemies de la raison; au contraire, elles comportent toujours des évaluations (plus ou moins conscientes et rapides) de ce qui est en train de se passer, et, comme le disent les philosophes contemporains, des antécédents cognitifs.

Certes, toute la littérature, du moins dans la culture occidentale, parle depuis toujours des passions humaines, mais rares sont les écrivains qui ont vraiment réfléchi à ce qu'on pourrait appeler: une théorie de la vie affective. Ces écrivains sont exactement ceux qui attribuent à la littérature une forme de connaissance. Cette question devient urgente pour la critique littéraire après le structuralisme et le poststructuralisme. Le fait même de s'interroger sur le rapport entre la littérature et la vérité représente une réaction contre toutes les tendances de la critique qui, dans la seconde moitié du $\mathrm{XX}^{\mathrm{e}}$ siècle, ont refusé non seulement l'idée que la littérature participe à l'entreprise de connaissance, mais que la connaissance ait une quelconque valeur. Dans un 
récent ouvrage, La connaissance de l'écrivain. Sur la littérature, la vérité, la vie, Jacques Bouveresse dénonce d'emblée deux types d'erreur, l'erreur esthétique et l'erreur formaliste ou postmoderne 9 . La première est enracinée dans la conviction qu'on ne peut pas aimer la littérature si on cherche en elle autre chose que le plaisir esthétique; la seconde se nourrit du mythe que rien n'existe en dehors du texte et que tout flotte dans une atmosphère de mystère. Bouveresse organise des réflexions croisées d'écrivains modernes et de philosophes et critiques contemporains en plusieurs petits chapitres et selon des thèmes qui émanent de la question principale, tels que «la connaissance de l'écrivain comme connaissance pratique » ou «l'inséparabilité du contenu et de la forme romanesques». Non seulement Musil, qui est largement étudié par Bouveresse, mais aussi Stendhal offrent une réflexion, à travers leurs œuvres diverses et leurs romans, exactement sur ces deux thèmes.

\section{Description dynamique}

Il est utile, pour débattre le problème de la littérature et de la vérité, de créer une taxinomie et de distinguer les buts exprimés directement ou indirectement par les écrivains et par leurs personnages - aussi bien que par les critiques, en sachant que les écrivains font inévitablement de la théorie littéraire. Bouveresse commente le réalisme et le naturalisme littéraires dans La connaissance de l'écrivain. Il cite Stendhal une fois, à propos de l'" amour exagéré pour la logique» dont l'écrivain se réclame pour récuser les attaques contre son style si souvent critiqué au XIX ${ }^{\mathrm{e}}$ siècle. Stendhal permet d'approfondir le problème du réalisme en littérature. On pourrait dire que les romanciers réalistes, ou considérés comme tels, se proposent de décrire le monde. Cette simplification veut indiquer que la description n'est pas le but de Stendhal, non pas qu'il refuse le monde, bien au contraire, mais parce qu'il pense le monde à travers la littérature. Décrire ne lui suffit pas, ou alors il lui faut une description dynamique qui soit déjà une explication.

Ce dynamisme se révèle de deux manières: la première est la description dynamique à proprement parler, à savoir la phrase nerveuse, mobile et rapide typique de Stendhal; la seconde consiste dans l'explicitation des hypothèses qui occupent l'esprit des personnages, souvent sous la forme du monologue intérieur. La description dynamique, ou la dynamique des émotions, est la solution de Stendhal pour rendre compte de la vie affective. C'est aussi pourquoi la littérature (romanesque) est le meilleur choix. Comme le dit Musil dans l'essai déjà cité, une science s'occupe d'un nombre fini de faits; la littérature en revanche s'occupe du réseau infini des motifs.

9. Voir Jacques Bouveresse La Connaissance de l'écrivain. Sur la littérature, la vérité, la vie, Marseille, Agone, 2008 , p. I3. Sur la vision postmoderne contre la science, ou les formes de "vériphobie contemporaine", voir Pascal Engel "La vérité malgré tout", in D. Khayat, N. Martin et A. Spire Maux pour maux, Paris, Le Bord de l'Eau, 200I, p. 63. 
Stendhal exprime sans cesse son intérêt pour la connaissance de la vie affective: ses romans seront la réalisation de ses soucis cognitifs. Dans ses lettres de jeunesse (dès I 804), dans ses récits et dans ses écrits sur l'art, Beyle insiste sur l'importance de la connaissance des passions et des sentiments, car cette connaissance est selon lui essentielle pour vivre avec le plus de bonheur possible, ou pour éviter le plus possible le malheur. Pendant plusieurs années, il écrit à sa sœur Pauline Beyle en lui recommandant de parvenir à ce savoir sur l'âme humaine. Pour cela, il lui suggère la bonne philosophie (Hobbes et Hume), la bonne littérature (Molière, Cervantès, Corneille), l'observation des comportements des gens dans les salons, les conversations avec ses amies, des questions sur ce qu'elles éprouvent et l'analyse de ce qu'ellemême éprouve ${ }^{10}$. Le travail d'analyse est essentiel.

En I 827 , Stendhal écrit à son ami italien Gian Pietro Vieusseux une longue lettre sur l'état de la philosophie à Paris. Il remarque que la philosophie n'est pas une seule science comme on le dit depuis le Moyen Âge, mais est composée de plusieurs sciences différentes: la logique, la métaphysique, laquelle se dédouble, avec d'un côté la réflexion sur Dieu et de l'autre la réflexion sur l'âme, enfin une quatrième et dernière branche qui intéresse le plus Stendhal et que nous pourrions appeler aujourd'hui: la psychologie ou la philosophie des émotions.

I. La logique ou les recherches sur la manière de ne pas se tromper en raisonnant, et l'art de ne pas se tromper fondé sur cette science.

2. L'explication et la connaissance de Dieu.

3. L'explication et la connaissance de l'âme.

4. L'explication de ce qui se passe dans le cœur de l'homme quand il éprouve une passion: l'ambition, la vengeance, etc. ${ }^{11}$

10. Voir Stendhal, Correspondance I, Paris, Gallimard, «Bibliothèque de la Pléiade», I968, p. I39 (à Pauline Beyle, 20 août I 804).

11. Stendhal, Correspondance II, Paris, Gallimard, "Bibliothèque de la Pléiade ", I967, p. I3 I-I 32 (lettre du 22 décembre I 827). Stendhal esquisse des taxinomies et des distinctions entre ce que nous appelons dans notre vocabulaire moderne, les passions, les humeurs, les dispositions et les émotions. Il explique à sa sœur: «Il y a des passions, l'amour, la vengeance, la haine, l'orgueil, la vanité, l'amour de la gloire.

Il y a des états des passions: la terreur, la crainte, la fureur, le rire, les pleurs, la joie, la tristesse, l'inquiétude.

Je les appelle états de passions, parce que plusieurs passions différentes peuvent nous rendre terrifiés, craignants, furieux, riants, pleurants, etc.

Il y a ensuite les moyens de passion, comme l'hypocrisie. Il y a ensuite les habitudes de l'âme; il y en a de sensibles, il y en a d'utiles. Nous nommons les utiles, vertus; les nuisibles vices. Vertus: justice, clémence, probité, etc., etc. Vices: cruauté, etc. Vertus moins utiles ou qualités: modestie, bienfaisance, bienveillance, sagesse, etc. Vices moins nuisibles ou défauts: fatuité, esprit de contradiction, mensonge, impertinence, mystère, timidité, distraction, etc.

Remarque que beaucoup de ces choses sont en même temps habitude de l'âme et défauts. Une passion peut rendre distrait, menteur; cela est bien différent d'avoir l'habitude de la 
Stendhal continue en rappelant que les Allemands ont beaucoup parlé de métaphysique et que ces mêmes discussions se poursuivent à Paris dans la voie tracée par «l'éloquent $\mathrm{M}$. Cousin »; il souhaite enfin que ces réflexions deviennent de plus en plus précises et "moins éloquentes " : alors "elles plairaient probablement davantage aux amateurs passionnés de la Vérité». Stendhal souligne ce mot, après avoir attaqué, comme d'habitude, le style peu clair et la confusion dans la pensée. Il exige la recherche de la clarté, la précision, l'abandon du bavardage prétentieux: c'est une valeur proprement cognitive, indispensable pour parvenir à la science.

Il n'y a pas de doute que le dernier élément dans la liste des sciences philosophiques est le plus important pour Stendhal et qu'il constitue le projet de ses romans. Combien de fois écrit-il, un peu partout, que l'intrigue dans les romans a peu de valeur, tandis que ce qui compte, c'est l'étude du cœur humain! La question se pose alors: pourquoi écrire des romans et non pas des traités? C'est la question même que se posera Musil. On peut répondre vite: parce que la littérature sollicite plus vivement l'imagination. Je vais élaborer cette réponse en reprenant le thème de la description dynamique chez Stendhal.

La description dynamique, dont Stendhal devient le maître insurpassable, consiste en l'amalgame compact, dans chaque phrase, entre des éléments qui viennent et du monde extérieur et du monde intérieur, ou entre percevoir, voir, sentir, comprendre, réagir, réfléchir, agir. C'est ce type d'amalgame que recherche la construction de la phrase, du paragraphe, ou même du chapitre. Cette construction a trompé beaucoup de lecteurs de Stendhal, dont le champion de la description «statique ", à savoir le théoricien du roman expérimental, Zola, qui condamne Stendhal pour son incapacité à présenter les lieux, les milieux et les rapports de cause à effet. Zola pense que la description scientifique du roman expérimental doit toujours rendre compte du milieu: "Nous estimons que l'homme ne peut pas être séparé de son milieu, qu'il est complété par son vêtement, par sa maison, par sa ville, par sa province; et, dès lors, nous ne noterons pas un seul phénomène de son cerveau, sans en chercher les causes ou le contrecoup dans le milieu ${ }^{12}$.»

Prenons l'exemple du célèbre chapitre du Rouge et le Noir qui est celuilà même auquel Zola fait référence dans son essai sur Stendhal ${ }^{13}$. Julien a

distraction, l'habitude de mentir, sujets de comédies traités par Regnard et Corneille. Pense à ces divisions de l'âme» (Correspondance I, Paris, Gallimard, Bibliothèque de la Pléiade, I968, p. I I 8, lettre à Pauline Beyle, juin I 804).

12. Voir Émile Zola«De la description», Euvres critiques I, Paris, Fasquelle, I898, p. I299.

13. Voir de Zola «Stendhal», Les Romanciers naturalistes, Euvres littéraires 2, Paris, Cercle du livre précieux, I978, p. 75-77. Voir aussi un texte indispensable de critique littéraire qui discute l'esthétique du miroir chez Stendhal et les raisons pour lesquelles il frustrait les attentes du naturalisme: Georges Blin, Stendhal et les problèmes du roman, Paris, José Corti, I953. 
décidé qu'il doit prendre la main de $\mathrm{M}^{\mathrm{me}}$ de Rênal et la serrer: c'est pour lui un devoir militaire, comme pour un soldat qui irait au combat. Il veut aller à ce combat, son devoir lui impose de séduire la dame chez laquelle il est instituteur. Le soir de la décision, Julien, $\mathrm{M}^{\mathrm{me}}$ de Rênal et $\mathrm{M}^{\mathrm{me}}$ Derville, une amie de celle-ci, sont dans le jardin de la maison des Rênal. Julien doit agir.

Enfin, comme le dernier coup de dix heures retentissait encore, il étendit la main, et prit celle de $\mathrm{M}^{\mathrm{me}}$ de Rênal, qui la retira aussitôt. Julien, sans trop savoir ce qu'il faisait, la saisit de nouveau. Quoique bien ému lui-même, il fut frappé, de la froideur glaciale de la main qu'il prenait, il la serrait avec une force convulsive, on fit un dernier effort pour la lui ôter, mais enfin cette main lui resta.

Son âme fut inondée de bonheur, non qu'il aimât $\mathrm{M}^{\mathrm{me}}$ de Rênal, mais un affreux supplice venait de cesser. Pour que $\mathrm{M}^{\mathrm{me}}$ Derville ne s'aperçût de rien, il se crut obligé de parler, sa voix alors était éclatante et forte. Celle de $\mathrm{M}^{\mathrm{me}}$ de Rênal, au contraire, trahissait tant d'émotion que son amie la crut malade et lui proposa de rentrer. Julien sentit le danger: «Si $\mathrm{M}^{\mathrm{me}}$ de Rênal rentre au salon, je vais retomber dans la position affreuse où j'ai passé la journée. J'ai tenu cette main trop peu de temps pour que cela compte comme un avantage qui m'est acquis ${ }^{14}$.»

La critique de Zola expose deux griefs majeurs contre Stendhal: le premier, trop de logique; le second, trop peu de précisions sur le milieu et aucune explication des rapports de cause à effet. Selon lui, si Stendhal avait été un vrai écrivain "réaliste» ou naturaliste avant la lettre, comme le sont Balzac, Flaubert et les Goncourt, il aurait dû bien décrire le lieu où l'épisode se passe, les vêtements des personnages, et surtout il aurait dû expliquer la raison pour laquelle la dame cède à la sensualité. Certes, l'idée suggérée par Zola que $\mathrm{M}^{\mathrm{me}}$ de Rênal laisse sa main dans celle de Julien à cause de la chaleur de l'été montre que l'écrivain déterministe n'est pas un bon lecteur de Stendhal. Zola n'a pas bien lu les paragraphes de ce chapitre du Rouge et le Noir, ni ceux des chapitres précédents, qui parlent de $\mathrm{M}^{\mathrm{me}}$ de Rênal, de sa sensibilité, de sa tendresse, etc. Mais la mauvaise lecture de Zola met en valeur une caractéristique du style de Stendhal: c'est qu'il impose à son lecteur un rythme si rapide dans l'analyse des émotions et des actions qu'on peut ne pas s'apercevoir des notations sur les lieux. On ne trouve pas de liste détaillée des objets du monde extérieur, mais seulement les éléments strictement nécessaires pour capter la contingence dans son devenir et structurer dans un faisceau mobile l'action, les sentiments et les évaluations (tout cet ensemble que Stendhal même appelle souvent: les mouvements de l'âme).

La bataille de Julien commence avec le son d'une cloche, qui dit donc bien l'heure de la scène; on sait qu'il y a un jardin et un salon, et encore, plus loin dans le long passage cité, du vent et le présage d'une tempête. Certes, un

14. Stendhal, Le Rouge et le Noir, Euvres romanesques complètes, Paris, Gallimard, «Bibliothèque de la Pléiade», 2005 , p. 397. 
écrivain comme Balzac aurait commencé par un long paragraphe sur le jardin, la couleur du ciel et l'atmosphère; par la suite il aurait pris une ou deux pages pour décrire toutes les expressions et les gestes d'un des deux personnages principaux, et encore une ou deux pages pour présenter l'autre personnage et faire entrer le lecteur dans la scène, en lâchant çà et là quelques considérations morales sur les êtres humains et leurs passions (et, bien sûr, sans jamais tomber dans la bêtise de dire que $\mathrm{M}^{\mathrm{me}}$ de Rênal cède à une sensualité provoquée par la chaleur). Le lecteur serait emporté par la fougue passionnée des adjectifs et il verrait un tableau bien coloré et contrasté de la scène de séduction. Stendhal court-circuite tout cela et le saisit dans un mouvement dynamique où l'extérieur et l'intérieur s'enchevêtrent, où les nuances des émotions font osciller les deux personnages entremêlés dans l'événement, la volonté s'enrobant de tendresse, la tendresse se muant en décision, les jugements se colorant de surprise, les mots devenant les signes de la peur, du plaisir, de l'excitation, du devoir. N'est-ce pas cela le bon "saint réel »? N'est-ce pas ainsi que les choses se passent dans la vie, que l'événement soit grand ou petit? Un tissu infini, rapide, continuellement changeant de relations et de motifs? Zola n'a rien vu, mais Proust, bien que n'étant pas un grand admirateur de Stendhal, a compris: «En un sens les beaux livres ajoutent aux événements une tranche d'âme coïncidente. Dans Le Rouge et le Noir, chaque action est suivie d'une partie de la phrase indiquant ce qui se passe inconsciemment dans l'âme, c'est le roman du motif ${ }^{15}$."

Quant au second élément de la dynamique de Stendhal, l'exposé des hypothèses, un grand nombre d'exemples peuvent illustrer les arguments et les contre-arguments esquissés par les personnages, les conjectures qui jaillissent dans leur esprit sans arrêt, à chaque fois qu'ils sont confrontés à un événement passé, en cours ou à venir. La scène de séduction entre Julien et $\mathrm{M}^{\mathrm{me}}$ de Rênal peut servir aussi à cette fin. Dans le peu de temps où se passe l'action de prendre et de serrer la main de $\mathrm{M}^{\mathrm{me}}$ de Rênal, Julien pense et se dit beaucoup de choses, tout en ressentant la main froide de la dame et le bonheur de la conquête. Les phrases hypothétiques montrent de manière éclatante les raisonnements ${ }^{16}$. La clause conditionnelle peut surgir en discours direct, comme dans le paragraphe qui suit le premier succès de Julien, dans les mots du jeune paysan: "Si Mme de Rênal rentre au salon, je vais retomber dans la position affreuse où j’ai passé la journée. »Et en discours indirect, lorsque le narrateur rapporte ce qui se passe dans la tête de Julien:

15. Marcel Proust, "Notes sur Stendhal», Essais et articles, in Contre Sainte-Beuve, Paris, Gallimard, la Pléiade, I971, p. 654.

16. Léon Blum, qui parle d'eux comme d'ergoteurs, a bien défini leur lucidité: «Les héros de Stendhal soliloquent [...]. Par une vue géniale, qui fait toute la fierté de ses héros et qui place l'analyse stendhalienne si fort au-dessus des psychologies communes, Stendhal, en effet, a conçu la curiosité critique et la clairvoyance de soi-même comme une dépendance du scrupule intime, du sentiment de l'honneur» (Léon Blum, Stendhal et le Beylisme, Paris, Albin Michel, I947, p. I4O-I4I). 
Au moment où $\mathrm{M}^{\text {me }}$ Derville renouvelait la proposition de rentrer au salon, Julien serra fortement la main qu'on lui abandonnait.

$\mathrm{M}^{\mathrm{me}}$ de Rênal, qui se levait déjà, se rassit en disant, d'une voix mourante:

— Je me sens, à la vérité, un peu malade, mais le grand air me fait du bien.

Ces mots confirmèrent le bonheur de Julien, qui, dans ce moment, était extrême: il parla, il oublia de feindre, il parut l'homme le plus aimable aux deux amies qui l'écoutaient. Cependant il y avait encore un peu de manque de courage dans cette éloquence qui lui arrivait tout à coup. Il craignait mortellement que $\mathrm{M}^{\mathrm{me}}$ Derville fatiguée du vent qui commençait à s'élever et qui précédait la tempête, ne voulût rentrer seule au salon. Alors il serait resté en tête-à-tête avec $\mathrm{M}^{\mathrm{me}}$ de Rênal. Il avait eu presque par hasard le courage aveugle qui suffit pour agir; mais il sentait qu'il était hors de sa puissance de dire le mot le plus simple à $\mathrm{M}^{\mathrm{me}}$ de Rênal. Quelque légers que fussent ses reproches, il allait être battu, et l'avantage qu'il venait d'obtenir anéanti ${ }^{17}$.

Si $\mathrm{M}^{\mathrm{me}}$ Derville était entrée dans le salon, alors «il serait resté en têteà-tête avec $\mathrm{M}^{\mathrm{me}}$ de Rênal ».

Dès le début de l'épisode, une série de considérations sont tressées à des remarques du narrateur, parfois pour ainsi dire dans le corps-à-corps du narrateur et du personnage, là où le style indirect libre brouille les voix et les perceptions: "Ces mots confirmèrent le bonheur de Julien, qui, dans ce moment, était extrême: il parla, il oublia de feindre, il parut l'homme le plus aimable aux deux amies qui l'écoutaient. Cependant il y avait encore un peu de manque de courage dans cette éloquence qui lui arrivait tout à coup. » Dans ce moment, cependant: voilà ces petits mots que le narrateur ne dit qu'en devenant son personnage même, car c'est Julien qui les pense dans sa lutte intérieure, dans sa guerre entre la volonté et la peur. Le narrateur rend le tempo de l'émoi par des ellipses, car les mots seraient bien plus lents que la saisie de l'épisode et l'enchaînement vertigineux des impressions. Le cependant qui suit la phrase sur la conversation aimable de Julien avec les deux dames brûle une étape: celle qui nous dirait que Julien continuait de juger son comportement selon le critère du courage militaire, critère de jugement qui ne peut pas appartenir au narrateur qui observe Julien dans son incapacité à jouir de la tendresse de l'amour. Un raccourci, mais parfaitement justifié par l'antécédent: Julien avait effectivement pensé qu'il fallait aller à l'amour comme à la guerre, en soldat courageux; d'où l'autocritique sur les possibles signes de lâcheté.

En effet, deux types de conjectures au moins interviennent dans les romans de Stendhal: les unes, très rapides au moment où l'événement se passe et que la sphère émotive est stimulée très rapidement. Un second type d'hypothèses, dont le roman est très riche, est en revanche la suite longue, bien explicitée, des raisonnements que le personnage accomplit lorsqu'il

17. , Stendhal Le Rouge et le Noir, op. cit., p. 398. 
étudie les émotions qu'il a éprouvées et les comportements qu'il a eus. Ce second type de conjectures forme les soliloques des personnages.

Les hypothèses auxquelles se livrent les personnages ou le narrateur sont de véritables investigations, comme on parle d'investigations philosophiques, qui permettent non seulement d'observer le réel, mais de comprendre le réel à travers le possible. Comme la ligne dont parle Musil et que l'on bouge "dans l'eau sans du tout savoir s'il y a une amorce au bout ", ces investigations essayent le réel. La valeur de connaissance de la littérature consiste en ces expériences de pensée. Ainsi, on peut répondre comme Musil à la question de savoir comment la littérature accède à la recherche de la vérité, en affirmant qu'elle le fait exactement comme les autres formes de recherche, par des hypothèses. La description est nécessaire, mais elle ne peut pas constituer un but en soi; elle n'est que le socle à partir duquel on élargit le champ du réel par l'imagination du possible. Il se trouve alors que le roman, plus qu'un traité, est semblable à la ligne que l'on peut traîner dans maintes directions possibles, avec plus d'intensité.

Les romans, le théâtre, la peinture, les arts ont affaire à la matière humaine: lorsqu'ils sont de qualité ils font bien plus que la décrire, que la documenter; ils l'expriment, l'analysent, l'approfondissent, agissent sur elle car ils frappent et aiguisent l'imagination.

\section{Entre parenthèses}

Plusieurs philosophes analytiques contemporains qui réfléchissent sur les émotions et les valeurs voient dans la littérature une forme de connaissance qui, comme le remarque Bouveresse, peut même «apporter à la philosophie pratique une contribution plus directement philosophique ${ }^{18}$. Martha Nussbaum parle de l'avantage de l'art, qui agrandit le pouvoir de l'imagination; Ronald de Sousa insiste lui aussi sur l'importance de l'imagination, indispensable dans tous les types d'échange humain ${ }^{19}$. Elle ne peut augmenter que sur la base de ce qui existe déjà. Dans les termes de Stendhal, c'est le miroir que l'on promène le long du chemin et qu'on ne pourrait pas concevoir sans le travail de l'imagination ${ }^{20}$.

18. Bouveresse, La connaissance de l'écrivain, op. cit., p. Io.

19. Voir Martha Nussbaum, Love's Knowledge, Oxford, Oxford University Press, I990. Peter Goldie parle d'une corrélation entre "gaining a new appreciation of aesthetic properties and gaining a new understanding of the world" in "Charley's World: Narratives of Aesthetic Experience ", Knowing Art, M. Kieran and D. Lopes (dir.), Oxford, Oxford University Press, 2006. Voir aussi Noël Carroll Engaging the Moving Image, New Haven, Yale University Press, 2003; Beyond Aesthetics: Philosophical Essays, Cambridge et New York, Cambridge University Press, 200I; Gregory Currie, The Nature of Fiction, Gregory Currie, Cambridge et New York, Cambridge University Press, I990; Arts and Minds, Oxford and New York, Clarendon Press, 2004. Un classique de critique littéraire sur le problème du roman et de la vérité: René Girard, Mensonge romantique et vérité romanesque, Paris, Hachette, I96I.

20. On voit que j'ai privilégié la thèse des vérités essentielles et que j'esquisse le travail de l'imagination comme expérience de pensée. Je n'explore pas ici la question de la fiction et du 
C'est ce travail que font les protagonistes des romans de Stendhal; ils s'expliquent à eux-mêmes les comportements des autres et leurs propres comportements, ils comparent une action, une situation, une phrase prononcée à d'autres possibles. Ils font des conjectures et ils se trompent; leurs intuitions et leurs gestes rapides sont le raccourci de raisonnements qui ont été faits ou qui seront faits; ainsi ils parcourent le chemin de la connaissance.

On peut alors déplier les couches d'ironie dans le calembour de SaintRéal: «Le roman est un miroir que l'on promène le long du chemin. » Cette même phrase revient presque identique plus loin dans le roman, sans la fausse attribution. Le roman représente ou exprime le monde réel, mais en mouvement: un écrivain réaliste arrêterait cette esthétique du miroir au simple reflet du miroir, alors que Stendhal veut que l'on se promène. Le réel pour les «saints " pédants de la description plate est un inventaire, mais il est «sacrosaint » pour ceux qui savent l'observer et le conjuguer à l'imagination.

Cette idée aide à interpréter une des interventions d'auteur les plus longues et les plus denses dans Le Rouge et le Noir. Dans un chapitre de la deuxième partie, le narrateur analyse les états d'âme contradictoires de Mathilde. Un soir à l'opéra, en écoutant la musique de Cimarosa, pour la première fois cette jeune femme noble, orgueilleuse, et qui veut toujours être forte, se déclare à elle-même son amour pour Julien, mais, conformément à son sens du devoir-être, elle pense dominer ses sentiments: "Le résultat de cette nuit de folie fut qu'elle crut être parvenue à triompher de son amour. » Le narrateur indique, avec une ironie tendre, la self-deception de Mathilde, qui croit ce qui n'est pas, et il ouvre alors une parenthèse, où la distinction entre auteur et narrateur n'est plus pertinente, pour ironiser sur la grande valeur de son siècle, l'intérêt économique.

(Cette page nuira de plus d'une façon au malheureux auteur. Les âmes glacées l'accuseront d'indécence. Il ne fait point l'injure aux jeunes personnes qui brillent dans les salons de Paris de supposer qu'une seule d'entre elles soit susceptible des mouvements de folie qui dégradent le caractère de Mathilde. Ce personnage est tout à fait d'imagination, et même imaginé bien en dehors des habitudes sociales qui parmi tous les siècles assureront un rang si distingué à la civilisation du XIX ${ }^{\mathrm{e}}$ siècle. Ce n'est point la prudence qui manque aux jeunes filles qui ont fait l'ornement des bals de cet hiver.)

Certes, Mathilde, âme sublime, est une créature «d'imagination » et non pas un personnage qui représente les «jeunes filles» avides de biens matériels. L'ironie satirique frappe le manque d'idéaux de ce siècle dont la civilisation "aura un rang si distingué ». Mais l'ironie se dirige aussi, peut-on dire, contre ces saints du réel qui réclament un miroir immobile réfléchissant

faire semblant, le make-belief. Voir Kendall L. Watson, Mimesis as Make-Believe. On the Foundations of the Representational Arts, Harvard, Harvard University Press, I993; et Gregory Currie The Nature of Fiction, Cambridge, Cambridge University Press, I990. 
les normes, les «habitudes sociales », la petitesse du monde. Ceux-là veulent exclure l'imagination du roman. Ils excluent, comme ceux qui s'attachent à l'hameçon, la possibilité de traîner la ligne dans l'eau, c'est-à-dire de faire penser, par le roman, à d'autres valeurs, plus nobles que celles du siècle, grâce, pour ainsi dire, à cette l'hypothèse vivante qu'est un personnage sublime, avec ses sentiments, ses contradictions et ses luttes intimes.

Après quelques pointes contre les jeunes filles à la chasse aux avantages de la fortune, Stendhal continue son discours entre parenthèses:

(Ce n'est point l'amour non plus qui se charge de la fortune des jeunes gens doués de quelque talent comme Julien; ils s'attachent d'une étreinte invincible à une coterie, et quand la coterie fait fortune, toutes les bonnes choses de la société pleuvent sur eux. Malheur à l'homme d'étude qui n'est d'aucune coterie, on lui reprochera jusqu'à de petits succès fort incertains, et la haute vertu triomphera en le volant. Eh, monsieur, un roman est un miroir qui se promène sur une grande route. Tantôt il reflète à vos yeux l'azur des cieux, tantôt la fange des bourbiers de la route. Et l'homme qui porte le miroir dans sa hotte sera par vous accusé d'être immoral! Son miroir montre la fange, et vous accusez le miroir! Accusez bien plutôt le grand chemin où est le bourbier, et plus encore l'inspecteur des routes qui laisse l'eau croupir et le bourbier se former.

Maintenant qu'il est bien convenu que le caractère de Mathilde est impossible dans notre siècle, non moins prudent que vertueux, je crains moins d'irriter en continuant le récit des folies de cette aimable fille.)

Ici, Stendhal glisse des personnages inventés à l'auteur lui-même: si celui-ci, sans se plier à servir une coterie, montre les vices des hommes, il sera accusé d'immoralisme. La parenthèse se ferme enfin sur la conclusion qui reprend le thème du caractère exceptionnel de Mathilde.

La difficulté de cette longue parenthèse tient à une mauvaise habitude de pensée qui oppose l'imagination au réel. C'est justement cette habitude que Stendhal veut changer. Le lecteur qui n'est pas assez rapide et qui s'attend à des inventaires ordonnés ou à des descriptions rangées se trouve déboussolé par ce discours entre parenthèses: il ne voit pas le lien entre la première partie, la seconde partie et la conclusion. Le parcours semble contradictoire. Il était d'abord question d'imagination avec Mathilde et il semble bien que, par le biais de l'ironie, l'auteur pense que l'imagination est une bonne chose. Par la suite, l'auteur prend le parti du miroir: l'écrivain doit rendre le réel, même et surtout s'il n'est pas glorieux. Par ce: «Eh, monsieur, un roman est un miroir qui se promène sur une grande route », l'auteur s'adresse directement à son lecteur et lance ses pointes ironiques contre les lecteurs et les écrivains opportunistes, non sans faire de l'auto-ironie sur le "pauvre» auteur. Enfin, il conclut sur Mathilde qui sort du moule social de son époque et est un produit de l'imagination.

Mais ce discours est parfaitement cohérent. Ce qui tient tout ensemble, c'est l'ironie qui s'infiltre et se propage, comme dans tout le roman, liquide, agile et variée avec ses formes différentes. La satire contre le siècle est présente 
dans chaque paragraphe du passage en question; l'ironie tendre s'adresse aux personnages chéris par leur créateur, Mathilde et Julien; et enfin, l'autoironie sur le "malheureux auteur» connaît deux sursauts: le premier, lorsqu'il se voit condamné pour son imagination; le second, lorsqu'il se voit attaqué pour sa sincérité et son manque d'opportunisme. C'est l'auteur qui fait ici sienne l'esthétique du miroir, la sainteté du réel; il réclame le droit et même le devoir de la littérature "à l'âpre vérité »: montrer le bourbier, la fange, ou, comme dirait Musil, l'obscène et le malsain ${ }^{21}$. Mais on ne parvient à aucune vérité sans solliciter l'imagination.

Stendhal pense que le roman doit parler de ce qui existe dans monde et que, du moment où il représente ses misères, il cesse d'être indécent ou immoral. Il estime que cette voie n'exclut pas que l'on développe, avec imagination, des situations utopiques. On peut ajouter que, légère comme un air d'opéra-bouffe, l'auto-ironie s'élève jusqu'à dire le plaisir de l'écrivain, plaisir d'imaginer et de raconter, de continuer « le récit des folies de cette aimable fille ". La conclusion sur Mathilde dans le passage entre parenthèses suggère encore l'avantage de l'imagination: imaginer des personnages nobles, qui ne se plient pas aux habitudes, aide à la réflexion de la morale pratique et à la compréhension de la différence entre les valeurs et les normes.

Stendhal arrive ainsi à résoudre, en termes strictement littéraires, l'opposition entre history et romance, ou entre réalisme et romantisme. Et, pour ce qui est du rapport entre la littérature et la connaissance, il esquisse sa vérité en littérature, son projet d'esthétique et d'éthique à la fois. La connaissance de l'homme intérieur par la littérature ne saurait exclure le plaisir pour l'écrivain et pour les lecteurs intelligents, les célèbres happy few de Stendhal, car, comme il est dit dans Vie de Henri Brulard: "Un roman est comme un archet, la caisse du violon qui rend les sons c'est l'âme du lecteur ${ }^{22}$.»

\section{Une question de style}

Le plaisir esthétique dont il est question ici n'a rien à voir avec l'erreur esthétique dont parle Bouveresse, à savoir la pruderie de ceux qui refusent que l'art soit un miroir de l'expérience vécue et des expériences humaines possibles. Il n'est pas non plus l'agrément qui peut accompagner comme un ornement une leçon morale. Stendhal ne pourrait pas souscrire à la thèse humaniste et moralisante du dulce et utile. La préface de Manon Lescaut (I728) peut servir à illustrer cette thèse qui envisage le plaisir esthétique comme complément de la mission éducative de la littérature.

21. Voir Musi «L'obscène et le malsain dans l'art», 1, Essais, op. cit., p. 25-3 I. L'occasion de cet article de I9I I a été la censure en Allemagne des carnets de Flaubert.

22. Stendhal Vie de Henri Brulard, Euvres intimes II, Paris Gallimard, Pléiade, I982, p. 699 . 
Il [le public] verra, dans la conduite de M. des Grieux, un exemple terrible de la force des passions [...] Les personnes de bon sens ne regarderont point un ouvrage de cette nature comme un travail inutile. Outre le plaisir d'une lecture agréable, on y trouvera peu d'événements qui ne puissent servir à l'instruction des mœurs; et c'est rendre, à mon avis, un service considérable au public, que de l'instruire en l'amusant [...].

Le contenu et la forme sont pour ainsi dire juxtaposés après coup, et non pas consubstantiels. La morale n'est pas pensée par Prévost comme connaissance de la vie morale: il ne s'agit donc pas d'une question cognitive. Surtout, il semble que les normes et les valeurs éthiques soient indifférenciées, alors que l'imagination des folies de Mathilde dans Le Rouge et le Noir pose justement cette différence. Prévost, non sans ironie, dit que l'on sert le public en lui montrant qu'il vaut mieux accepter les normes pour se défendre des effets néfastes des passions. Or, chez Stendhal, le plaisir esthétique ne saurait se partager des contenus que la littérature traite: il est dans l'inséparabilité de la forme et du contenu.

Une dernière conséquence de l'investigation sur les pouvoirs de la littérature à partir de Stendhal est la réhabilitation de la grande littérature contre l'idée moderne et postmoderne que tout est texte, et que les textes "canoniques" sont nécessairement coupables, car ils dérivent d'une construction sociale de la qualité littéraire qui, au lieu d'abriter des valeurs intrinsèques, résulte des structures de pouvoir. Stendhal, avec Musil, raisonne à la hausse et non pas à la baisse: ils savent que, pour donner une connaissance de la vie vécue et inventer l'homme intérieur, un bon roman doit trouver un style à la hauteur de ses ambitions.

Lorsque Ronald de Sousa écrit aujourd'hui que ce qui compte en littérature n'est pas "how probable, but how vividly imaginable something is ${ }^{23}$ ", il souligne la valeur de l'imagination dans la fiction et, par l'adverbe vividly, il condense une foule de significations littéraires: vivid est ce qui est vivant, convaincant, frappant sur le plan de la forme et du contenu, ce qui présente dans un ensemble quelque chose qui saisit à la fois l'intellect et les émotions. C'est le style qui est vivid.

Le style, c'est ce qui permet d'arriver, au travers de multiples efforts, à l'inséparabilité de la forme et du contenu. La preuve des efforts est dans les journaux et la correspondance de ces écrivains, ainsi que dans leurs critiques d'œuvres littéraires. Musil signale souvent que ce à quoi il doit résister, c'est sa tendance à s'enliser dans la théorie; et Stendhal, même s'il a pu dicter en trente-deux jours La Chartreuse de Parme, n'en finit plus d'accumuler dans les marges de ses manuscrits son: For me, signe tangible de sa course vers l'idéal de son style, le vivid qui contient la vie et l'imagination.

23. De Sousa, "The Art of the Possible in Life and in Literature», in M. E. Reicher, J. C. Marek (dir.), Experience and Analysis,Vienne, 2005 p. $35 \mathrm{I}$. 
Il n'est pas surprenant alors que l'inséparabilité de la forme et du contenu se dessine chez ces deux écrivains de la même manière: dans le renoncement au bavardage, à la forme qui embellit et éloigne de la vie. Stendhal, qui détestait les phrases rhétoriques, boursouflées et "pleines de petites faussetés de Chateaubriand ", aimait le style du code civil. Qu'une langue sobre, précise, sans superlatifs, aille droit aux yeux, au cœur et au cerveau du lecteur. Musil partage cet idéal, présent déjà dans Les Désarrois de l'élève Törless:

Je me rappelle encore le principe qui me guidait en écrivant Törless: m'en tenir au maximum de concision, refuser toute image qui n'ajoutait rien à l'idée, écarter toute pensée, si fort que je pusse y tenir, qui ne s'intégrât pas naturellement dans le cours de l'action. Le principe de la ligne droite comme plus court chemin entre deux points ${ }^{24}$.

Même s'il n'a écrit que quelques remarques sur Stendhal, Musil veut arriver à un style vivid et concis qui s'apparente à celui de Chesterton et de Stendhal:

La lecture de Chesterton m'a directement aidé, hier, à trouver l'expression à la fois souple et libre d'une idée. Ses exemples frappants, vigoureux avaient agi sur moi. [...] Ce n'est pas sans rapport avec l'exemple de Stendhal lisant le code de Napoléon avant de se mettre au travail [...] Je précise que l'influence de Chesterton avait agi dans le sens de la «sobriété»: écrire sobrement! J'ai cet avertissement sous les yeux depuis des semaines, mais je l'oublie parfois; Chesterton n'a fait que me donner le geste ${ }^{25}$.

Il n'y a aucun doute, il faut frapper le lecteur avec la force d'une idée. C'est dans sa célèbre lettre à Balzac, lequel, dans un article très élogieux sur $\mathrm{La}$ Chartreuse, avait émis quelques critiques sur le style, que Stendhal évoque l'inspiration qui lui viendrait du Code civil et son horreur pour le style de Chateaubriand. Il s'excuse auprès du maître, mais il ne veut pas changer sa manière d'écrire: "Souvent je réfléchis un quart d'heure pour placer un adjectif avant ou après son substantif. Je cherche à raconter: I) avec une idée, 2) avec clarté ce qui se passe dans un cœur $^{26}$.» Toute sa vision de la littérature est dans cette phrase, dans la clarté qu'elle peut apporter à la connaissance du cœur.

24. Musil, «Testament II», Journaux II, traduction de Philippe Jaccottet, Paris, Seuil, I98 I, p. 700 .

25. Musil «Cahier 3I (I930-I936)», Journaux II, op. cit., p. 33 I-332.

26. Stendhal, Correspondance, III, op. cit., p. 395, (lettre à Balzac, I6 octobre, I840). Stendhal souligne.

Cet article a été rédigé dans le cadre du Projet «Affective Dynamics and Aesthetic Emotions» du Swiss National Center of Competence in Research in Affective Sciences de l'Université de Genève. 